\title{
Bemerkungen zur Polarität der Angiospermen-Eizelle
}

\author{
Von Kurt StefFen \\ Aus dem Botanischen Institut der Universität Marburg (Lahn)
}

(Z. Naturforschg. 1, 533-538 [1946]; eingegangen am 23. Juli 1946)

\begin{abstract}
Die verschieden zur Schwerkraft orientierten Samenanlagen von Impatiens glanduligera Royle geben Anlaß dazu, die Schwerkraft als Polarität induzierende oder die korrelative Beeinflussung hemmende oder fördernde Kraft auszuschließen. Die Schwerkraft hat keinen Einfluß auf die Stellung des Kernes und der ersten Teilungswand in der befruchteten Eizelle und auf die weitere Entwicklung des Embryos. Zellsprossungen, wie $\mathrm{H}$ a b e r l and t sie beschreibt, konnten als Abnormität in 1,8\% aller Fälle beobachtet, aber nicht in seinem Sinne gedeutet werden.

Die Wirkung der Polarität wird in der Richtung der ersten Teilungswand und in dem differenten Teilungsmodus der beiden ersten Blastomeren gesehen. Abnormitäten in den ersten Pro-Embryo-Stadien lassen erkennen, daß bereits in der befruchteten Eizelle die Teilungsmodi der beiden ersten Kerne determiniert sind. Dieser Befund wird in dem Sinne gedeutet, daß die an sich isotrope Eizelle unter Polaritätswirkung ausgerichtet und die totipotenten Blastomeren in ihrer Entwicklungsrichtung festgelegt werden.

Die Frage nach der strukturellen oder korrelativ bedingten Polarität kann mit heutigen Mitteln nicht entschieden werden. Korrelative Beeinflussung wird aus den Literaturangaben über abnorme Fortpflanzungszustände und aus dem Verhalten des sekundären Endospermkernes und der weiteren Entwicklung des Embryos geschlossen. Eine Verknüpfung von morphologischer Form und funktioneller Polarität der befruchteten Eizelle scheint nicht berechtigt.
\end{abstract}

Ctellen wir das Bekannte und Allgemeingültige Doran: die Eizelle der Angiospermen ist meist heteropolar gebaut. Die morphologische Polarität der Eizelle kommt zum Ausdruck in der ungleichen Plasmaverteilung und der Lage des Zellkernes. Die scheitelständige, exzentrische Lage des Zellkernes bedingt die Lage der Kernspindel und bei quer gestellter Teilungswand die ungleiche Größe der Tochterzellen ${ }^{1}$. In der Richtung der Kernspindel und damit auch in der Richtung der eingeschalteten Teilwand sehen wir eine funktionelle, also physiologische Wirkung der Polarität. „Die Richtung der ersten Teilungsspindel ist bestimmend für die Bipolarität der Pflanze, die aus der Eizelle hervorgeht" ${ }^{\text {2 }}$.

Hiermit aber sind auch unsere Kenntnisse dieses „noch immer völlig rätselhaften Gebietes der Cytologie“ erschöpft.' „Denn auf keinem anderen Gebiet der Morphologie ist unsere Unkenntnis des Wesentlichen so offenkundig, wie gerade hier", so

1 G. H a berl andt, S.-B. preuß. Akad. Wiss., Physik.-math. Kl. 1928, S. 450.

${ }^{2} \mathrm{~K}$. S chnarf, Vergleichende Cytologie des Geschlechtsapparates der Kormophyten, Berlin 1941, S. 202. referiert F. Weber ${ }^{3}$ die sich mit den Problemen der Eizelle befassende Arbeit von R. S ou èges ${ }^{4}$. Und $\mathrm{S}$ o u è ge s selber beklagt den Mangel an geeigneten Beobachtungen, der eine Deutung und Wertung so schwer und unsicher macht. Die hier nun mitgeteilten Beobachtungen an einem jahrelang studierten Objekt sollen das Beobachtungsmaterial vergrößern und zu einer möglichst klaren Fragestellung führen. Mehr kann aus den genannten Gründen zur Zeit nicht erwartet werden.

Voraussetzung für jede Arbeit ist eine klare Nomenklatur. Es ist zwischen unbefruchtetem und befruchtetem Ei oder Zygote zu unterscheiden. Die Pole der Eizelle sind mit ihrer Lagebezeichnung: mikropylarer oder basaler Pol (pôle inférieur, végétatif oder radiculaire nach Soùge $\mathrm{s}^{5}$ ) und chalazaler oder apikaler Pol (pôle supérieur, germinatif oder caulinaire nach $\mathrm{S}$ o u è ge $\mathrm{s}$, „Scheitel der Eizelle"nach Ha ber land $\mathbf{t}^{\mathbf{1}}$ ) deutlich gekennzeichnet. Die Längsachse verbindet beide Pole, sie

3 Protoplasma 23, 293 [1935].

${ }^{4}$ La cellule embryonnaire. Actualités scientifiques et industrielles, Paris, 208 [1935].

5 La segmentation. Deuxième fascicule. Actualités scientifiques et industrielles, Paris 318 [1936]; Bull. Soc. bot. France 2, 45 [1933]. 
ist zugleich Wachstumsachse. Sie wäre mit E. K ü ster $^{6}$ heteropolar zu nennen. Der Ausdruck: „heteropolar" ist dem indifferenten ,bipolar", der zusammen mit dem Ausdruck: ,tetrapolar" die Lageverhältnisse im Embryosack bezeichnet, vorzuziehen. Bei den Angiospermen sind drei Eizelltypen zu unterscheiden: 1 . der birnförmige, 2 . der zylindrische und 3. der abnorm vergrößerte (Oeufs piriformes [Typ 3], cylindriques or en tube [Typ 4] und géantes, anormales [Typ 5] nach S ou ège $\mathrm{s}^{4}$ ).

Die hier zu besprechende, morphologisch heteropolare Eizelle von Impatiens glanduligera Royle befindet sich in einem bipolaren Embryosack, dessen Polarität erst im 8-kernigen, bereits zellig differenzierten Embryosack nach Ausbildung der Vakuole deutlich wird (vergl. dazu die das Gleiche beweisenden Abbildungen 5 und 6 von K. V.O. D a h lg ren ${ }^{7}$ ).

Die Bedeutung der Vakuolisation für die Polarisation des Embryosackes wird durch die Beobachtung einer so späten Ausbildung der Vakuole problematisch. F. L. R u tg e s $^{8}$ setzt Vakuolisation und Polarisation des Embryosackes gleich, während A. Chiarugi ${ }^{9}$ in der Vakuolisation nur einen Wachstumsvorgang der Makrospore sieht, der durch die nachfolgende Kernteilung erst zur Polarisation führt. Wenn man die Bipolarität des Embryosackes in der differenten Ausbildung der beiden Vierergruppen sieht, muß man sagen, daß im hier beschriebenen Fall die Degeneration der Antipoden bereits vor Ausbildung der Vakuole, die Differenzierung des Eiapparates von drei morphologisch gleichwertigen Zellen zur Eizelle und zu den Synergiden aber erst nach Ausbildung der Vakuole erfolgt. Das Absterben der Antipoden braucht nicht als polarisationsbedingt aufgefaßt zu werden, wohl aber die Ausbildung des Eiapparates. So können das späte Auftreten der Vakuole und die bereits degenerierenden Antipoden nicht als Gegenbefund für eine polarisierende Wirkung der Vakuolisation aufgefaßt werden. Ob die Vakuolisation etwa im Sinne von F. W. We n ${ }^{10}$ ein Potentialgefälle bedingt oder vergrößert und damit zur Polarität im Embryosack führt, muß offen bleiben. Eine Polaritätsumkehr wurde trotz des reichen Untersuchungsmaterials von 2500 Samenanlagen nie beobachtet.

\footnotetext{
${ }^{6}$ Die Pflanzenzelle, Jena 1935.

7 Svensk Bot. Tidskr. 28, 103 [1934].

8 Ann. Jard. bot. Buitenzorg 33, 1 [1923].

9 Nuovo giorn. bot. Ital. N.S. 34, 1 [1927].

10 J. wiss. Bot. 76, 528 [1932].
}

Die anatropen, apotropen und hängenden Samenanlagen erfahren während ihrer Entwicklung eine zweite Drehung, die die Mikropyle aus der Ebene: Funiculus/Fruchtknotenachse seitlich herausdreht und dabei die Samenanlagen oft rechtwinklig zu dieser Ebene stellt, worauf bereits H.S a u er ${ }^{\mathbf{1 1}}$ und K. V. O. D a hlg ren ${ }^{7}$ hingewiesen haben. Die Embryonen entwickeln sich in jedem Fall normal und vollständig. Auf einem Fruchtknotenlängsschnitt, wie ihn die Abbildung 1 zeigt, beobachtet man also neben längs geschnittenen in günstigen Fällen auch genau quer geschnittene Samenanlagen. Diese Tatsache ermöglicht es also, etwas über den Einfluß der Schwerkraft an den durch die Natur selbst schon so verschieden orientierten Samenanlagen auszusagen; diese Tatsache hat mich vor allem bewogen, meine Beobachtungen hier niederzulegen.

Die Wirkung der Schwerkraft könnte als polaritätsinduzierende Kraft oder, falls die Polarität korrelationsbedingt ist (vergl. die zusammenfassende Behandlung der Polaritätsfragen bei N.P. $\mathrm{Krenke}{ }^{1}$ ), als korrelationsfördernder oder -hemmender Faktor und so die Ausbildung wie die Auswirkung der Polarisation fördernde oder hemmende Kraft in Erscheinung treten. Damit offenbart sich zugleich die Schwierigkeit des Gesamtproblems.

Betrachten wir zunächst die morphologische Polarität der Eizelle: die voll entwickelte Eizelle von Impatiens glanduligera ist birnförmig, jedoch nicht radiär-symmetrisch, da ihr basales Ende einem schief aufgesetzten Kegelstumpf gleicht, der durch die Anheftung an die Embryosackwand eine Abplattungsfläche bekommen hat (Abb. 2). Der Zellkern liegt meist bis auf $1 \mu$ dem apikalen Ende genähert und wird auch häufig der Zellwand völlig anliegend gesehen. Die große Vakuole liegt am basalen Pol der Zelle.

Der Zellkern erfährt in der wachsenden, sich differenzierenden Eizelle eine Verlagerung von der Zellmitte zum apikalen Pol der Zelle. Diese Zellkernverlagerung kann aktiv oder passiv erfolgt $\operatorname{sein}^{13}$. Da der Zellkern stets apikal liegt, wie auch die Samenanlage zur Schwerkraft orientiert sein möge, sind als Schwerkraftwirkungen ausgeschlossen: bei der Annahme einer passiven Kernverlage-

11 Planta 19, 417 [1933].

12 Wundkompensation, Transplantation und Chimären bei Pflanzen, Berlin 1933, S. $301 \mathrm{ff}$.

${ }^{13} \mathrm{~T}$ ischler, G., Allgemeine Pflanzenkaryologie. Handbuch d. Pflanzenanat., Berlin 1934, Bd. I/2, 1. Hlft. 
rung die Lageveränderung durch Änderung des spezifischen Gewichtes des Kernes in einem spezifisch leichteren Medium, und als aktive Wirkung die geotaktische Kernwanderung. Passive Kernverlagerung durch eine sich bildende Vakuole muß zum mindesten für die $5 \%$ aller beobachteten Fälle ausfallen, wo der Zellkern ohne Vakuolenbildung doch am apikalen Pol lag. Die die Verlagerung des Kernes unterstützende Wirkung der Vakuolenbildung wird damit auch in diesem Fall nicht geleugnet, ebenso wenig die Möglichkeit einer sekundären Unterstützung durch cytoplasmatische Strömungen; doch muß dabei bemerkt werden, daß dann allerdings 'die topographisch fixierte Lage des Zellkernes einer besonderen Erklärung bedürfte.

Da keine durch die Beobachtung sich ergebenden Anhaltspunkte für eine aktive, lokomotorische Kernwanderung auf amöboider Grundlage ,zum Ort der stärksten Plasmaanhäufung“" im Sinne von K. Linsbauer ${ }^{14}$ vorliegen, bliebe als wahrscheinlichste Deutung die passive Kernverlagerung durch sich ändernde Oberflächenspannungsverhältnisse, deren Mechanik noch unklar ist ${ }^{6}$.

Eine finale Deutung der apikalen Lage des Zellkernes gibt $\mathrm{H}$ a be $\mathrm{r}$ landt ${ }^{1}$. Er macht für die von ihm bei den befruchteten Eizellen von Ornithogalum nutans, Alisma plantago, Eucomis undulata und Oenothera Lamarckiana beobachteten Zellsprossungen und für ähnliche in der Literatur beschriebene und abgebildete Zustände die exzentrische, der sprossenden Wand genäherte Lage des Zellkernes verantwortlich und glaubt, daß die hier beobachtete Wirkung bei befruchteten Eizellen verbreitet und besonders bei Monokotylen häufig sei.

$\mathrm{Da}$ in der von Haberlandt beschriebenen Zellsprossung bereits eine Wirkung der Polarität zu sehen ist, bin ich diesen Dingen nachgegangen. In $1,8 \%$ aller befruchteten Eizellen ( 7 Fälle bei 391 darauf untersuchten in 6 von 66 untersuchten Fruchtknoten, das sind 9\% aller Fruchtknoten) habe ich eine bruchsackartige Vorstülpung feststellen können (Abb. 7). Die Lage des Zellkernes

\footnotetext{
14 Akad. Wiss. Wien, Math.-nat. Kl. Abt. I, 141, 1 [1932].
}

war in diesen Fällen nie besonders der vorgestülpten Wandung genähert. Der etwa im Sinne H a berland ts zu machende Einwand, daß sich die Kerne dieser Zellen bereits im zweiten Stadium, im Stadium des Abrückens von der Zellwand, befunden haben könnten, wird durch die Feststellung entkräftet, daß in $10 \%$ aller befruchteten Eizellen die Zellkerne der apikalen Zellwandung fest anliegen. Wäre die blasenförmige Vorstülpung nun durch die Lage des Zellkernes bedingt, so müßte der Prozentsatz der Zellsprossung weit höher sein. Würde das Membranwachstum durch
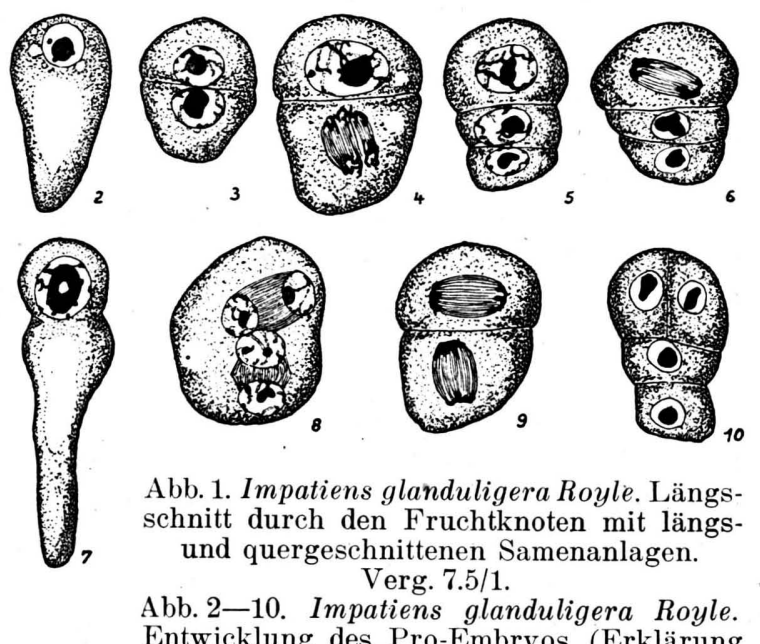

Abb. 1. Impatiens glanduligera Royle. Längsschnitt durch den Fruchtknoten mit längsund quergeschnittenen Samenanlagen. Verg. 7.5/1.

Abb. 2-10. Impatiens glanduligera Royle. Entwicklung des Pro-Embryos (Erklärung im Text). Vergr. 500/1.

die scheitelständige Lage des Zellkernes bedingt, so müßte es zur Zeit des stärksten Membranwachstums in der unbefruchteten Eizelle auftreten. Die Zellsprossung wird aber nur in der befruchteten Eizelle beobachtet, wie auch $\mathrm{Hab}$ er land t bereits festgestellt hat. Das Wachstum der befruchteten Eizelle aber ist, wie ich an anderer Stelle ${ }^{15}$ bereits dargetan habe, im Vergleich zum Wachstum der unbefruchteten, bereits völlig differenzierten, also befruchtungsreifen Eizelle sehr gering. Die beobachteten bruchsackartigen Vorstülpungen können also im Falle von Impatiens glanduligera nicht als durch die scheitelständige Lage des Zellkernes bedingt angesehen werden. Eine Erklärung für diese abnormen Wachstumsvorgänge kann ich nicht geben, da weder das Endosperm noch das Endothel einen abnormen Befund zeigten. Ich muß noch ergänzend bemerken, daß zuweilen auch der

${ }^{15}$ Untersuchungen an Impatiens glanduligera Royle. Dissertat. Marburg 1944. 
Kern von einer Einschnürung betroffen wurde, die augenscheinlich passiv durch die Formveränderung der befruchteten Eizelle bedingt war. In dem hier behandelten Fall vermag ich also nicht in der beschriebenen Wachstumsabnormität eine besondere Form morphologischer Polarität oder gar die physiologische Auswirkung einer strukturellen Polarität zu sehen.

Wir kommen somit zu der wesentlichen Frage, ob die Polarität strukturell oder korrelativ bedingt sei. Oder mit anderen Worten, ob eine indifferente Zelle durch eine einmalige Induktion in eine nunmehr irreversibel, strukturell polarisierte übergeht oder ob durch eine permanente, korrelative Beeinflussung eine reversible Polarität in der Zelle erzeugt wird. Für einzellige, niedere Pflanzen ist die Induktion der Polarität erwiesen. Von der Angiospermen-Eizelle müssen wir allerdings mit K. Lin sbau e ${ }^{16}$ sagen: „Dort, wo die Zygote sich nicht isoliert, sondern ihre Entwicklung im Zusammenhange mit den Geweben der Mutterpflanze beginnt, wird sich überhaupt kein Zeitpunkt finden, in dem sie völlig frei von Nachbarschaftswirkung wäre, die ihre Struktur von vornherein influenzieren könnte." Da sich diese korrelative Beziehung durch isolierte Kultur der Eizellen nicht aufheben läßt, ist diese Frage für die Angiospermen-Eizelle nicht zu entscheiden.

Eine induktive Wirkung der Schwerkraft kommt wegen der verschiedenen Orientierung der Samenanlagen bei Impatiens glanduligera nicht in Betracht. S o u è g e $\mathrm{s}$ hält eine Erwerbung der Polarität während der Reifezeit der Zygote für möglich. Die wenigen sicheren Befunde über eine generative Parthenogenesis und die etwas zahlreicheren für eine somatische lassen kein sicheres Urteil zu, ob die Polarität bereits in der unbefruchteten Eizelle bestanden hat oder nicht. Die bekannt gewordenen Fälle von Apogamie ${ }^{17}$ lassen meines Erachtens zusammen mit den spärlichen Befunden über die Parthenogenesis eher auf eine kontinuierliche korrelative Beeinflussung schlieBen. Die korrelative Beeinflussung kann durch die Lage der Eizellen und durch einen einseitigen Nähr- und Wuchsstoffstrom gegeben sein. Die Befunde an Adventivembryonen scheinen dafür zu

16 Rückdifferenzierung als Voraussetzung ontogenetischer Entwicklung. Flora 118/119, 346 [1925].

${ }_{17} \mathrm{~K}$. S c h n a r f, Embryologie der Angiospermen. Handbuch d. Pflanzenanat., Berlin 1929, Bd. X/2; Vergleichende Embryologie der Angiospermen, Berlin 1931 . sprechen. Da sich bei Impatiens glanduligera derartig abnorme Fortpflanzungserscheinungen nicht zeigen, kann hier kein Beitrag zu dieser Frage geliefert werden.

Wahrscheinlich gemacht wird eine korrelativ bedingte Polarität im Embryosack durch das Verhalten des sekundären Embryosackkernes. Die beiden Polkerne können in jeder erdenklichen Lage miteinander verschmelzen, wie in einer noch unveröffentlichten Arbeit dargetan ist. Der sekundäre Embryosackkern selbst ist also anfangs sicher nicht polarisiert, und doch ist die eingeschaltete Wand immer eine Querwand (vergl. die Abbildungen bei K. V. O. D a hlg re ${ }^{7}$ ). Die Polarität muß also entweder durch das Plasma des Embryosackes strukturell bedingt sein, oder aber das Cytoplasma des Embryosackes unterliegt der gleichen korrelativen Beeinflussung wie die Eizelle, was ich für wahrscheinlicher halte. Für eine korrelative Beeinflussung scheint mir ferner die Tatsache zu sprechen, daß die Ausbildung der Kotyledonen nicht durch eine bestimmte Teilwand prädeterminiert wird und unabhängig von den ersten Meridionalwänden in der oberen Embryozelle (ca) ist ${ }^{15}$.

Das Wesen der Polarität, das wir in einem stofflichen oder energetischen Gefälle sehen ${ }^{18}$, wird durch diesen unentschiedenen Fragenkomplex nicht berührt. Das stoffliche und energetische Gefälle kann im Falle einer strukturellen wie einer korrelativ beeinflußten Polarität gleichermaßen vorhanden sein.

$\mathrm{Zu}$ prüfen bleibt die Auswirkung der Polarität und ihre Beeinflußbarkeit durch äußere Einflüsse, wobei stets $\mathrm{zu}$ bedenken ist, daß hemmende oder fördernde Beeinflussung bereits das Korrelationsgefüge treffen kann und unter Umständen erst sekundär in der Polaritätswirkung in Erscheinung treten kann. Eine Deutung der Befunde muß also überaus vorsichtig sein und kann erst an Hand eines großen Tatsachenmaterials erfolgen. Das aber fehlt zur Zeit.

Die Auswirkungen der Eizell-Polarität zeigen sich: 1. in der Richtung der ersten Teilungswand und 2. in dem differenten Teilungsmodus der beiden ersten Zellen.

Die erste Teilwand ist, wie bei fast allen gegliederten Embryonen der Angiospermen, so auch

18 E. Bünning, Die Physiologie des Wachstums und der Bewegungen. Lehrbuch der Pflanzenphysiologie II, Berlin 1939, S. 52. 
bei Impatiens glanduligera, senkrecht zur Längsachse der Eizelle gestellt (Abb. 3). Diese erste Teilwand, so sagt man, entscheidet über die Polarität der Pflanze, welchen Anteil nun auch die beiden ersten Zellen am Embryo i.e.S. haben mögen ${ }^{2}$. In Wirklichkeit ist diese Teilungswand ja nur der sichtbare Ausdruck der in der Eizelle bereits vorhandenen Polarität. Die Richtung der ersten Teilungswand ist bei Impatiens glanduligera, wie auch die Samenanlagen zur Schwerkraft orientiert sein mögen, stets gleich. Auch das Eindringen des Spermakernes, das ja von verschiedenen Seiten her erfolgen kann, hat auf die Teilungsrichtung keinen Einfluß. Es gibt überhaupt nur sehr wenige gegliederte Embryonen, bei denen die Richtung der ersten Teilungswand von quer zu schräge variiert, und noch viel weniger Embryonen, bei denen die erste Teilungswand eine Längswand ist, wie ich bereits an anderer Stelle ausführlich dargetan habe ${ }^{15}$. Bei diesen wenigen abnormen Formen zeigt sich meist eine mehr oder minder weit gehende Störung im Aufbau, so z. B. bei Scabiosa succisa, bei der $\mathrm{S}$ o u è g e $\mathrm{s}^{18}$ keinerlei Regelmäßigkeit mehr feststellen konnte, und so im Extrem bei den Peperomia-Arten, wo kein gegliederter und polarisierter Embryo mehr entsteht ${ }^{20}$. Es wird uns nicht wundern, daß bei diesen Embryonen, bei denen uns der innere Bauplan der Pflanze gestört zu sein scheint, auch die Polarität labil ist, und die ersten Teilungsschritte abnorm verlaufen. Aus dem Verlauf dieser ersten Teilungswände nun weitgehende Schlüsse über die Polarität der befruchteten Eizelle ziehen zu wollen, erscheint verfrüht, da es sich zumeist um nicht genauer verfolgte Gelegenheitsbeobachtungen handelt.

An dieser Stelle ist es nun angebracht, etwas über die Richtung der Teilungswand in einer Zelle allgemein $\mathrm{zu}$ sagen. $\mathrm{S}$ o u è g e s ${ }^{21}$, der sich ja vor allem mit den Fragen der Embryogenie befaßt hat, glaubt an die Gültigkeit allgemeiner Teilungsgesetze, wie sie von $\mathrm{O}$. Hertwig und $\mathrm{S}$ a c h s aufgestellt wurden, und die er nun durch innere und äußere Faktoren modifiziert findet, und nach dem Grade der Abweichung von diesen Teilungsgesetzen mißt er auch die Ursprünglichkeit der Embryonalentwicklung. Für ihn stellt

19 C. R. hebd. Séances Acad. Sci. 204, 292 [1937].

20 D. H. C a m p b e l l, Ann. Botany 13, 626 [1899]; Ber. dtsch. bot. Ges. 17, 452 [1899]; D.S. J o hn s on, Bot. Gaz. 30, 1 [1900]; G. C. F i s h e r, Bull. Torr. Bot. Club 41, 137221 [1914]. sich also die Kernspindel in die Richtung der größten Plasmamasse, und die nächste Teilwand hat das Bestreben, senkrecht zur vorigen zu stehen. G. Tischler lehnt die S a ch sche Regel und den Erklärungsversuch von Errera $a b$, für ihn ist die Richtung der Teilungswand allein von der Kernspindel abhängig, wenngleich wir heute auch der Micellierung nach $\mathrm{H}$. Z i e g e $\mathrm{n} \mathrm{p} \mathrm{e} \mathrm{c} \mathrm{k}{ }^{23}$ einen gewissen richtenden Einfluß bei der Teilwandstellung zubilligen wollen. G. $\mathrm{T}$ is chle ${ }^{22}$ (S. 151) lehnt weiterhin allgemein eine „feste Polarisierung“ im Kern sowohl für die Anlage wie für die Richtung der Kernspindel ab und glaubt, daß die Lage der Kernspindel in den „Bauplan des ganzen Organismus“ eingeordnet sein müsse. $\mathrm{Zu}$ diesem Bauplan aber gehört meines Erachtens auch die Polarität. E. K ü s te $\mathrm{r}^{\boldsymbol{B}}$ hält es für möglich, daß die Richtung der Kernspindel durch die Richtung der Plasmateilchen bedingt sei, und G. $\mathrm{H} \mathrm{a}$ b e r l a n d $\mathrm{t}^{24}$ glaubt, daß sie sich in die Diffusionsrichtung seiner Wundhormone einstelle. Die Möglichkeit einer Kernpolarisation wird von T is chle r bei seiner Besprechung der Fucaceen-Zygoten zugegeben. Vom Standpunkte der Wentschen Polaritätstheorie wäre eine Kernpolarisation durchaus möglich, da ja auch der Kern seiner Lage nach sich in einem elektrischen Feld befände und dessen Gesetzen unterworfen wäre. Zusammenfassend ist also zu sagen, daß wir nichts über die Kräfte wissen, die die Stellung der Kernspindel bedingen. Sicher ist, daß in unserem Fall die erste Teilwand quer gestellt ist, wie auch die befruchtete Eizelle zur Schwerkraft orientiert ist. Die Teilungsrichtung in den beiden neu gebildeten Zellen ist verschieden. Die untere (cb) teilt sich stets quer (Abb. 4) und meist vor der sich längs teilenden oberen Zelle (ca) (Abb. 6). Die beiden Tochterzellen (ca und cb) sind also bereits in bezug auf ihre Teilungsrichtung funktionell differenziert. Es resultiert auf diesem Wege eine Tetrade en T (Abb. 10), wie S o u è g e $\mathrm{s}$ diese Stellung nennt. Die zunächst offensichtlich größere Teilungsgeschwindigkeit von ca wird bei einer Zwischenstadien außer acht lassenden, zusammenfassenden Betrachtung ${ }^{15} \mathrm{zu}$

${ }^{21} \mathrm{La}$ segmentation. Premier fascicule. Actualités scientifiques et industrielles, Paris 266 [1935].

22 Allgemeine Pflanzenkaryologie. Handb. d. Pflanzenanat., Berlin 1942, Bd. I/2, 2. Hlft., S. 221.

23 Protoplasma 36, 514 [1942].

24 Wundhormone als Erreger von Zellteilungen. Beitr. allgem. Bot. 2, 1 [1921]. 
einer in beiden Blastomeren gleichmäßigen; so wundert es uns also auch nicht, daß in Einzelfällen ( $5 \%$ aller untersuchten Stadien: Abb.3-6) auch tatsächlich eine gleichzeitige Teilung der beiden Zellen $\mathrm{zu}$ beobachten ist (Abb.9). Diese Abweichung von einer sonst herrschenden Regel der Teilungsgeschwindigkeit (Loi des nombres ou loi des vitesses nach So ù̀ ge ${ }^{25}$ ) erfährt eine weitere Abwandlung dadurch, daß die Bildung der ersten Teilwand unterbleibt und nun in beiden Eibezirken die Kernteilung gleichzeitig und nach demselben Teilungsmodus wie bisher erfolgt (Abb. 8). Somit hat sich das Gesetz über die Stellung (Loi des dispositions nach $\mathrm{S}$ o u è g e s) der vier ersten Blastomeren auch unter abnormen Verhältnissen als beherrschend erwiesen. Dieser Fall ist sehr selten. Er ist nur in 2\% aller Fälle realisiert. Der einzige mir aus der Literatur bekannte Fall, in dem die befruchtete Eizelle freie Kernteilung erfährt, ist von F. L. Ru tg e r s ${ }^{8}$ bei Moringa oleifera heschrieben worden. Freie Kernteilung in der unbefruchteten Eizelle findet sich nach G. Tis chle ${ }^{26}$ bei Ficus carica und nach S. Kusano bei Gastrodia elata ${ }^{27}$. Diese spärlichen Angaben in der Literatur beweisen das seltene Vorkommen solcher abnormen Zustände.

Die Tochterkerne haben also auch im gemeinsamen Zellraum beim teilweisen Aussetzen sonst herrschender Gesetze ihre spezifisch festgelegte Teilungsrichtung. Will man nicht eine differente Polarisierung der Tochterkerne annehmen, so muß man zu dem Schluß kommen, daß in der befruchteten Eizelle zum mindesten die Potenzen $\mathrm{zu}$ diesen differenten Teilungsmodi lokalisiert sein müssen. Das würde im strengen Sinne bereits eine Anisotropie des Eies bedeuten, doch ist $\mathrm{zu}$ bedenken, daß die Segmentierung noch nicht gleichbedeutend mit einer Differenzierung ist, wenn sie auch oft eine solche einleitet.

Meines Erachtens ist die Polarität in dem Gesamtbauplan der Pflanze einbegriffen und nicht gesondert zu werten; sie wird sich in vielen Fällen nicht als Einzelfaktor absondern lassen. Ihre Wirkung auf die Segmentierung kann zunächst

\footnotetext{
25 Les lois du développement. Actualités scientifiques et industrielles, Paris 522 [1937].

${ }_{26}$ Jb. wiss. Bot. 52, 1 [1912].

27 J. Coll. Agric., Tokyo, 6, 7 [1915].

28 Bull. Soc. bot. France 57, 47 [1910].

29 J. G. Ha ll, Bot. Gaz. 33, 214 [1902].
}

nicht bewiesen, sondern nur wahrscheinlich gemacht werden. Sie tritt als richtender Faktor bei der Verteilung der im Ei noch latenten Potenzen auf. Die Totipotenz der gebildeten Blastomeren wird durch sie nicht aufgehoben, jedoch in ihrer Auswirkung beschränkt. Fehlt die richtende Kraft der Polarität, so tritt die volle Totipotenz der Blastomeren zutage. So kommt S o ù̀ g es auf Grund seiner Befunde bei Anemone Pulsatilla ${ }^{28}$, wo sich cb statt ca zum Embryo entwickelt, zu. der Utberzeugung, daß das $\mathrm{Ei}$ in bezug auf die Lokalisation der Potenzen isotrop sei. Als weitere Stütze seiner Ansicht führt er die Fälle an, wo aus verschiedenen Blastomeren des bereits mehrzelligen Pro-Embryos mehrere Embryonen auf dem Wege echter Polyembryonie entstehen können, so z. B. bei Limnocharis emarginata ${ }^{29}$, Erythronium $^{30}$ und Vincetoxicum nigrum ${ }^{31}$.

Im Falle von Anemone Pulsatilla könnte man von einer Polaritätsumkehr und in den anderen Fällen von einem Aufhören jeder Polaritätswirkung sprechen. Meines Erachtens schließen sich hier die schiefen, spiraligen und unregelmäßigen Embryonen nach S o u è g e ${ }^{5}$ (ungegliederte Em-

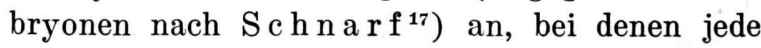
Polaritätswirkung ausgeschaltet scheint. Daß diese richtende Wirkung auch an sonst sich normal entwickelnden Embryonen gestört sein kann, sieht man an den von $\mathrm{S}$ o u è g e s ${ }^{32}$ beschriebenen polymorphen Formen.

Eine Kausalverknüpfung der beobachteten heteropolaren Form der Eizelle und der funktionellen Polaritätswirkungen ist nicht möglich. Die Lage des Zellkernes kann nur dann die Bildung zweier ungleich großer Zellen bewirken, wenn wir annehmen, daß die erste Teilwand unter dem Einfluß der Polarität quer gestellt ist. Nach den bisherigen Befunden ist kein Grund vorhanden, anzunehmen, daß die Eizelle ganz allgemein die Bildung zweier ungleich großer Zellen anstrebt, wie $\mathrm{H}$ aberlandt ${ }^{1}$ es meint. Das bei den einzelnen Pflanzen so überaus verschiedene Schicksal der unteren Tochterzelle spricht dagegen.

${ }^{30}$ E. E. J e f f r e y, Ann. Botany 9, 537 [1895]; P. Gu e r i n, C. R. hebd. Séances Acad. sci. 191, 1369 [1930].

31 L. Guigna rd, Mém. Acad. R. sci. Inst. de France, 2. sér. 57, 1 [1922].

${ }^{32}$ La différenciation. Deuxième fascicule. Actualités scientifiques et industrielles, Paris, 381 [1936]. 